\title{
Dynamical study of 2D and 3D barred galaxy models
}

\author{
T. Manos ${ }^{1,2}$ and E. Athanassoula ${ }^{2}$ \\ 1 Center for Research and Applications of Nonlinear Systems (CRANS), \\ Department of Mathematics, University of Patras, GR-26500, Greece. \\ thanosm@master.math.upatras.gr \\ 2 Laboratoire d'Astrophysique de Marseille (LAM), Observatoire Astronomique \\ de Marseille-Provence (OAMP), 2 Place Le Verrier, 13248 Marseille, Cédex \\ 04, France. \\ lia@oamp.fr
}

Summary. We study the dynamics of 2D and 3D barred galaxy analytical models, focusing on the distinction between regular and chaotic orbits with the help of the Smaller ALigment Index (SALI), a very powerful tool for this kind of problems. We present briefly the method and we calculate the fraction of chaotic and regular orbits in several cases. In the 2D model, taking initial conditions on a Poincaré $\left(y, p_{y}\right)$ surface of section, we determine the fraction of regular and chaotic orbits. In the 3D model, choosing initial conditions on a cartesian grid in a region of the $\left(x, z, p_{y}\right)$ space, which in coordinate space covers the inner disc, we find how the fraction of regular orbits changes as a function of the Jacobi constant. Finally, we outline that regions near the $(x, y)$ plane are populated mainly by regular orbits. The same is true for regions that lie either near to the galactic center, or at larger relatively distances from it.

\section{Introduction}

The dynamical evolution of galactic systems depends crucially on their orbital structure and in particular on what fraction of their orbits is regular, or chaotic. Thus to permit further studies, it is essential to be able to distinguish between these two types of orbits in a manner that is both safe and efficient. This is not trivial and becomes yet more complicated in systems of many degrees of freedom. A summary of the methods that have been developed over the years can be found in [1].

In the present paper we use a method based on the properties of two deviation vectors of an orbit, the "Smaller ALingment Index" (SALI) [2]. It has been applied successfully in different dynamical systems $[2,3,4,5,6,7$, $8,9,10,11,12]$, frequently also under the name Alignment Index (AI) [13, $14,15,16,17]$ and has been shown to be a fast and easy to compute indicator 
of the chaotic or ordered nature of orbits. We first recall its definition and we then show its effectiveness in distinguishing between ordered and chaotic motion by applying it to a barred potential of 2 and 3 degrees of freedom. Recently, a generalization of the SALI, the "Generalized ALignment Index" (GALI) was introduced by Skokos et al. (2007) [18], which includes the full set of the $k$ initially linearly independent deviation vectors of the system to determine if an orbit is chaotic or not.

\section{Definition of the Smaller ALigment Index - SALI}

Let us consider the $n$-dimensional phase space of a conservative dynamical system, which could be a symplectic map or a Hamiltonian flow. We consider also an orbit in that space with initial condition $P(0)=\left(x_{1}(0), x_{2}(0), \ldots, x_{n}(0)\right)$ and two deviation vectors $\boldsymbol{w}_{1}(0), \boldsymbol{w}_{2}(0)$ from the initial point $P(0)$. In order to compute the SALI for a given orbit one has to follow the time evolution of the orbit itself, as well as two deviation vectors $\boldsymbol{w}_{1}(t), \boldsymbol{w}_{2}(t)$ which initially point in two different directions. At every time step the two deviation vectors $\boldsymbol{w}_{1}(t)$ and $\boldsymbol{w}_{2}(t)$ are normalized by setting:

$$
\hat{w}_{i}(t)=\frac{\boldsymbol{w}_{i}(t)}{\left\|\boldsymbol{w}_{i}(t)\right\|}, \quad i=1,2
$$

and the SALI is then computed as:

$$
S A L I(t)=\min \left\{\left\|\hat{w}_{1}(t)+\hat{w}_{2}(t)\right\|,\left\|\hat{w}_{1}(t)-\hat{w}_{2}(t)\right\|\right\} .
$$

The properties of the time evolution of the SALI clearly distinguish between regular and chaotic motion as follows: In the case of Hamiltonian flows or dimensional symplectic maps with $n \geq 2$, the SALI fluctuates around a non-zero value for regular orbits $[2,3]$. In general, two different initial deviation vectors become tangent to different directions on the torus, producing different sequences of vectors, so that SALI does not tend to zero but fluctuates around positive values. On the other hand, for chaotic orbits SALI tends exponentially to zero. Any two initially different deviation vectors tend to coincide in the direction defined by the nearby unstable manifold and hence either coincide with each other, or become opposite.

\section{The model}

A 3D rotating model of a barred galaxy can be described by the Hamiltonian function:

$$
H=\frac{1}{2}\left(p_{x}^{2}+p_{y}^{2}+p_{z}^{2}\right)+V(x, y, z)-\Omega_{b}\left(x p_{y}-y p_{x}\right) .
$$

The bar rotates around its $z$ axis, while the $x$ axis is along its major axis and the $y$ axis is along its intermediate axis. The $p_{x}, p_{y}$ and $p_{z}$ are the 

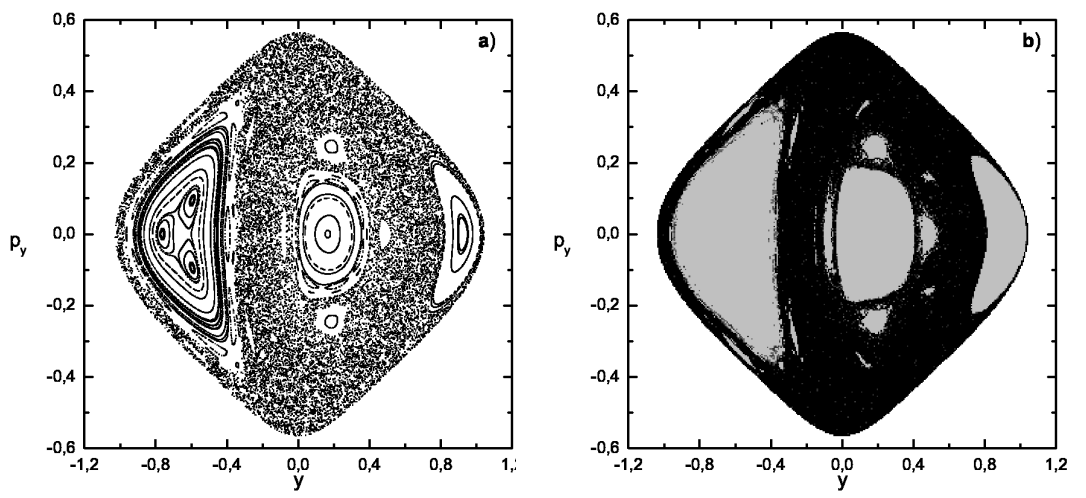

Fig. 1. Agreement between the results of the Poincaré surface of section (PSS) and SALI. a) The Poincaré surface of section for the 2D Ferrers model and $H=-0.335$. b) Regions of different values of the SALI for 50,000 initial conditions on the $\left(y, p_{y}\right)$ plane integrated up to $t=5000$ for the same value of the Hamiltonian. The light grey colored areas correspond to regular orbits, while the dark black ones to chaotic. Few gray and dark gray colored points correspond to "sticky" orbits. Note the excellent agreement between the two methods as far as the gross features are concerned, as well as the fact the SALI can easily pick out small regions of instability which the PSS has difficulties detecting.

canonically conjugate momenta. Finally, $V$ is the potential, $\Omega_{b}$ represents the pattern speed of the bar and $H$ is the total energy of the system in the rotating frame of reference (Jacobi constant). The corresponding equations of motion are:

$$
\begin{aligned}
& \dot{x}=p_{x}+\Omega_{b} y, \quad \dot{y}=p_{y}-\Omega_{b} x, \quad \dot{z}=p_{z}, \\
& \dot{p_{x}}=-\frac{\partial V}{\partial x}+\Omega_{b} p_{y}, \quad \quad \dot{p_{y}}=-\frac{\partial V}{\partial y}-\Omega_{b} p_{x}, \quad \dot{p_{z}}=-\frac{\partial V}{\partial z} \text {. }
\end{aligned}
$$

The equations of the evolution of the deviation vectors and the calculation of the SALI are given by the corresponding variational equations.

The potential $V$ of our model consists of three components:

1. A disc, represented by a Miyamoto potential [19]:

$$
V_{D}=-\frac{G M_{D}}{\sqrt{x^{2}+y^{2}+\left(A+\sqrt{z^{2}+B^{2}}\right)^{2}}},
$$

where $M_{D}$ is the total mass of the disc, $A$ and $B$ are the horizontal and vertical scale lengths and $G$ is the gravitational constant. 
2. A bulge, which is modeled by a Plummer sphere whose potential is:

$$
V_{S}=-\frac{G M_{S}}{\sqrt{x^{2}+y^{2}+z^{2}+\epsilon_{s}^{2}}}
$$

where $\epsilon_{s}$ is the scale length of the bulge and $M_{S}$ is its total mass.

3. A triaxial Ferrers bar, the density $\rho(x)$ of which is:

$$
\rho(x)=\left\{\begin{array}{cc}
\rho_{c}\left(1-m^{2}\right)^{2} & , m<1 \\
0 & , m \geq 1
\end{array},\right.
$$

where $\rho_{c}=\frac{105}{32 \pi} \frac{G M_{B}}{a b c}$ is the central density, $M_{B}$ is the total mass of the bar and

$$
m^{2}=\frac{x^{2}}{a^{2}}+\frac{y^{2}}{b^{2}}+\frac{z^{2}}{c^{2}}, \quad a>b>c>0,
$$

with $a, b$ and $c$ being the semi-axes. The corresponding potential is:

$$
V_{B}=-\pi G a b c \frac{\rho_{c}}{n+1} \int_{\lambda}^{\infty} \frac{d u}{\Delta(u)}\left(1-m^{2}(u)\right)^{n+1},
$$

where

$$
\begin{gathered}
m^{2}(u)=\frac{x^{2}}{a^{2}+u}+\frac{y^{2}}{b^{2}+u}+\frac{z^{2}}{c^{2}+u}, \\
\Delta^{2}(u)=\left(a^{2}+u\right)\left(b^{2}+u\right)\left(c^{2}+u\right),
\end{gathered}
$$

$n$ is a positive integer (with $n=2$ for our model) and $\lambda$ is the unique positive solution of:

$$
m^{2}(\lambda)=1
$$

outside of bar $(m \geq 1)$ and $\lambda=0$ inside the bar.

This model has been used extensively for orbital studies [20, 21, 22, 23, 24, 25] and we will refere to it hereafter as the Fererrs model. We adopt the following values of parameters: $G=1, \Omega_{b}=0.054\left(54 \frac{K m}{\mathrm{sec} \cdot K p c}\right), a=6, b=1.5, c=0.6$, $A=3, B=1, \epsilon_{s}=0.4, M_{B}=0.1, M_{S}=0.08, M_{D}=0.82$. The units we use, are: $1 \mathrm{kpc}$ (length), $1 \mathrm{Myr}$ (time) and $2 \times 10^{11} M_{\odot}$ solar masses (mass). The total mass $G\left(M_{S}+M_{D}+M_{B}\right)$ is set to be equal to 1 .

\section{Results in the 2D and 3D Ferrers model}

The 2D Ferrers model is a subcase of the general $3 \mathrm{D}$ one and it can be described by the Hamiltonian equation (3) by setting $\left(z, p_{z}\right)=(0,0)$ :

$$
H=\frac{1}{2}\left(p_{x}^{2}+p_{y}^{2}\right)+V(x, y)-\Omega_{b}\left(x p_{y}-y p_{x}\right) .
$$



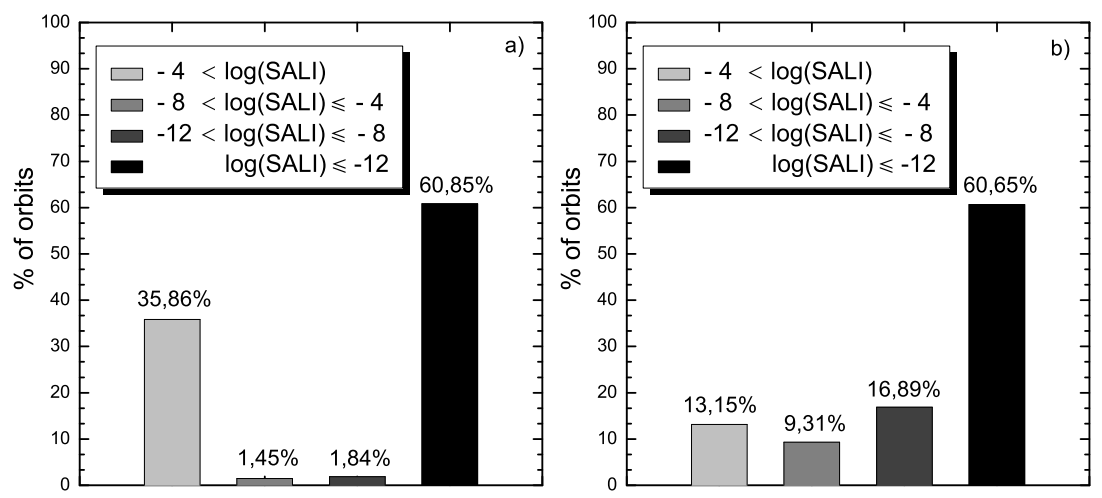

Fig. 2. a) Percentages of regular (light gray bar), chaotic (black bar) and sticky orbits (two intermediate bars - gray and dark gray bars) for the 2D Ferrers model from a mesh of 50,000 orbits on the $\left(y, p_{y}\right)$ plane of Fig. 1 and final time of integration $t=5000$. b) The percentages for $3 \mathrm{D}$, using 50,000 orbits and the same classification. The initial conditions are taken as described in the text.

Fixing $H=-0.335, x=0$, we chose 50,000 initial conditions on the $\left(y, p_{y}\right)-$ plane of the Poincaré surface of section (PSS), while $p_{x}=H\left(x, y, p_{y}\right)$ and we calculated the final values of the SALI for $t=5000$. We were able to detect very small regions of instability that can not be visualized easily by the PSS method. In Fig. 1, on the left panel, we have plotted the PSS for this Hamiltonian value. On the right panel we attributed to each grid point a color according to the value of the SALI at the end of the evolution. The light grey color corresponds to regular orbits and to the areas that host them while the black color represents the chaotic ones. The intermediate colors between the two extremes represent the so-called "sticky" orbits, i.e. orbits whose nature is chaotic but need more time to show their behavior (weak-chaotic orbits). The distinction between them is done by measuring SALI at $t=5000$ : orbits with $\mathrm{SALI} \leq 10^{-12}$ correspond to strongly chaotic, orbits with SALI $>10^{-4}$ to regular, while orbits with $10^{-8}<\mathrm{SALI} \leq 10^{-4}$ and $10^{-12}<\mathrm{SALI} \leq 10^{-8}$ are "sticky". In Fig. 2a we present the percentages of orbits from this PSS, according to this classification. We find that $\simeq 37,3 \%$ of the orbits on this PSS are regular.

For the 3D case of the model we used a sample of 50,000 orbits, equally spaced on a cartesian grid in the $\left(x, z, p_{y}\right)$ space, with $x \in[0.0,7.0], z \in$ $[0.0,1.5]$ and $p_{y} \in[0.0,0.45]$, while $\left(y, p_{x}, p_{z}\right)=(0,0,0)$. In this way, we attempted to create initial conditions that could support the bar. These orbits could be parented mainly by the $x_{1}$ tree, i.e. the $x_{1}$ family and the $x_{1} v_{i} i=1, \ldots$ families that bifurcate from it and extend vertically well above the disc region [21]. Although these initial conditions cover all the available energy interval, they are not spread uniformly over it. Note also that a few of these have an 

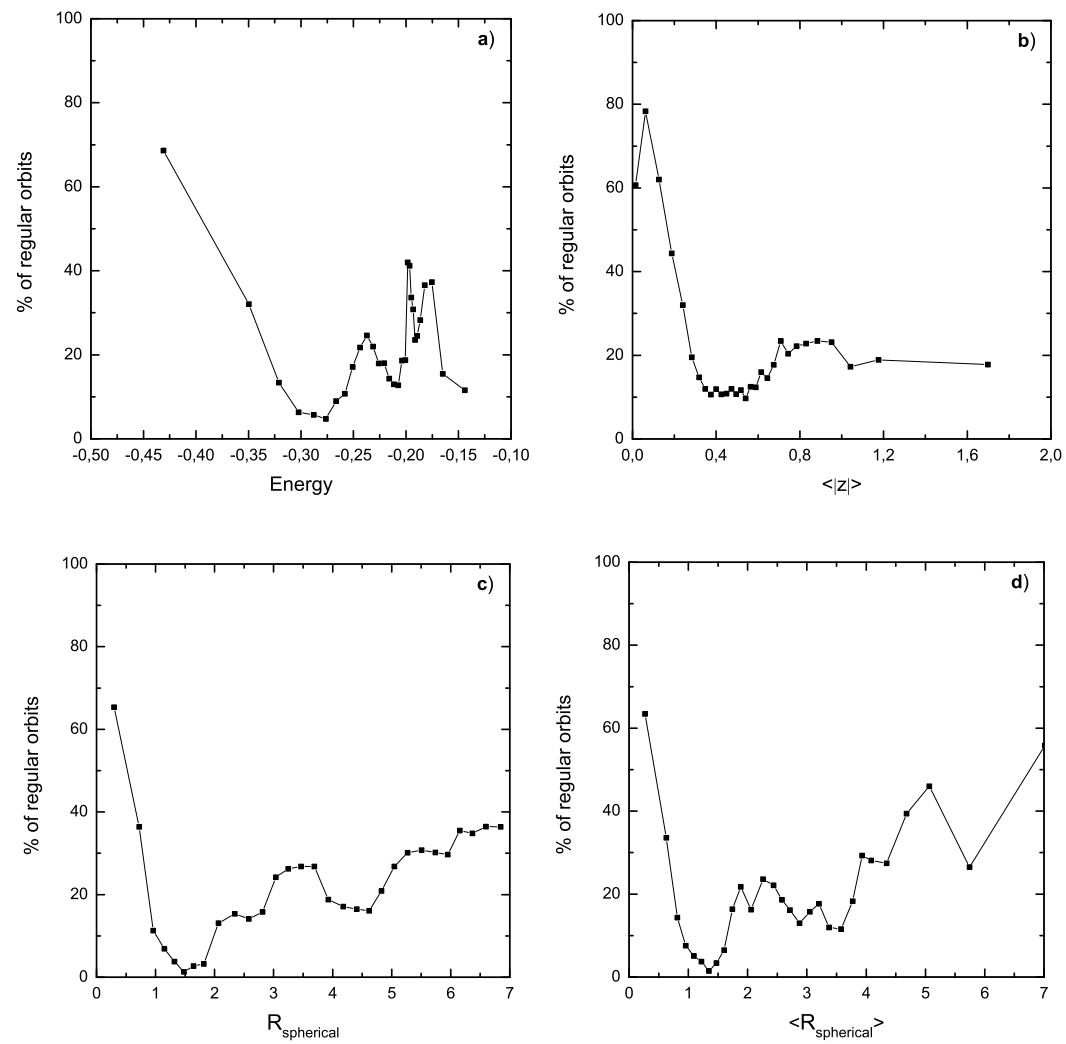

Fig. 3. Percentages of regular orbits as a function of: the Jacobi constant (panel a), the absolute value of their $z$ coordinate, $\langle|z|>$, meaned over their orbital evolution (panel b), their initial spherical radius, $R_{\text {spherical }}$ (panel c) and the spherical radius, $<R_{\text {spherical }}>$, meaned over their motion (panel d).

energy value beyond the escape energy, and we dismiss them. These, however, constitute less than $0.5 \%$ of the total, so that they influence very little our study and the statistics. In Fig. $2 \mathrm{~b}$ we present the corresponding percentages of regular, chaotic and sticky orbits. We find that, using these initial conditions and parameters, the phase space of the model is dominated by chaotic orbits, since $77.54 \%$ of them are chaotic.

In order to check the variation of the percentages of regular and chaotic orbits as the energy of the model varies, we did the following: We first sorted the energy values for all the initial conditions. Then, we created 30 energy intervals containing equal number of orbits, since our way of giving initial conditions does not imply their uniform distribution in the total energy interval. In every energy interval we calculated the percentages of regular and chaotic orbits, considering as chaotic all the orbits with $\mathrm{SALI}<10^{-8}$. In Fig. 3a we show the 
percentage of regular orbits in each energy interval, as a function of the mean energy in that interval. Generally, the percentage of regular orbits decreases as the energy increases, but before and after the escape energy (where the Jacobi constant value is $H \simeq-0.20$ ) there are two peaks. This non-monotonic behavior is related to the appearance or disappearance of stable periodic orbits in the phase space and the size variation of the stability regions around them.

We also attempted to explore the way that regular and chaotic orbits are distributed along the $z$-direction of the configuration space. Following the evolution of each orbit, we calculated the mean of the absolute value of their $z$ coordinate $(<|z|>)$. Then, we divided the available $<|z|>$-interval in 30 slices with equal number of orbits in each one of them. This restriction gives us better samples for the estimation of the percentages, implying at the same time that these slices are not equally sized necessarily. For every slice separately we calculated the fraction of regular orbits and in Fig. 3b we plot these percentages as a function of the $\langle|z|>$ in that slice. It reveals that the slices 'near' the $(x, y)$-plane $(<|z|><0.35)$ contain mainly 'regular' orbits. Contrarily, slices for larger values of $z$ host mainly chaotic motion.

Furthermore, we looked at these percentages as a function of the initial spherical radius $\left(R_{\text {spherical }}\right)$ and the mean spherical radius $\left(<R_{\text {spherical }}\right\rangle$, meaned over the evolution). Again, dividing in 30 slices the total range of the $R_{\text {spherical }}$, in a similar manner with the $\langle|z|>$, we calculated the percentages of regular orbits. We plot this for every slice, as a function of the mean $R_{\text {spherical }}$ of that slice. We see that the fraction of regular orbits decreases strongly with increasing $R_{\text {spherical }}$ up to $R_{\text {spherical }}<1.5$ where it reaches a minimum, while for $1.5<R_{\text {spherical }}<7$ this percentage starts increasing gradually. This result is in good agreement with the results in Fig. 3d, where the horizontal axis corresponds to the value of the spherical radius meaned over time during the evolution.

\section{Conclusions}

We used SALI to study the dynamical behavior of Hamiltonian models of 2D and 3D barred galaxies. We found that in both cases there is a significant amount of chaotic orbits. In the 2D model, we were able to chart a subspace of the phase space, to identify rapidly even tiny regions of regular motion and measure their percentages. In the 3D model, apart from computing the global percentages of regular and chaotic orbits, we calculated these percentages as a function of the energy and found that low values of the energy are mainly dominated by 'regular' orbital motion. We also followed the distribution of the chaotic and regular orbits in the configuration space and we found that orbits which lie near the $(x, y)$-plane with relatively small mean deviations in $z$-direction are generally regular. Finally, we monitored the variation of their percentages as a function of their initial spherical radius and their mean 
spherical radius. We find that the fraction of regular orbits is dominant in regions near the center, as well as at relatively larger distances from it.

\section{Acknowledgments}

T. Manos was partially supported by the "Karatheodory" graduate student fellowship No B395 of the University of Patras, the program "Pythagoras II" and the Marie Curie fellowship No HPMT-CT-2001-00338. We acknowledge financial support from grant ANR-06-BLAN-0172.

\section{References}

1. Contopoulos, G., 2002, Order and chaos in dynamical astronomy, SpringerVerlag, Berlin

2. Skokos Ch. 2001, J. Phys. A: Math. Gen., 34, 10029

3. Skokos Ch., Antonopoulos Ch., Bountis T. and Vrahatis M., 2003, Prog. Theor. Phys. Suppl., 150, 439

4. Skokos Ch., Antonopoulos Ch., Bountis T. and Vrahatis M., 2004, J. Phys. A, 37, 6269

5. Panagopoulos P., Bountis T. and Skokos Ch., 2004, J. Vib. \& Acoust., 126, 520

6. Antonopoulos Ch. and Bountis T., 2006, Physical Review E, 73, 056206

7. Antonopoulos Ch., Bountis T. and Skokos Ch., 2006, Int. J. Bif. Chaos, Vol. 16, No. 6, 1777

8. Bountis T. and Skokos Ch., 2006, Physics Letters A, 358, 126

9. Manos T. and Athanassoula E., 2005, SF2A-2005: Semaine de l'Astrophysique Francaise, Edited by Casoli F., Contini T., Hameury J.M. and Pagani L., Published by Edp-Sciences, Conference Series, 631

10. Manos T. and Athanassoula E., 2005, $5^{\text {th }}$ International Cosmology Conference: The Fabulous destiny of galaxies: Bridging past and present, eds. Le Brun V., Mazure A., Arnouts S., Burgarella D., Frontier Group

11. Manos T. and Athanassoula E., 2006, AIP Conference Proceedings, Recent Advances in Astronomy and Astrophysics: $7^{\text {th }}$ International Conference of the Hellenic Astronomical Society, Vol. 848, 662

12. Manos T. Skokos Ch., Athanassoula E. and Bountis T., 2007, $19^{\text {th }}$ Panhellenic Conference/Summer School "Nonlinear Science and Comlexity", Thessaloniki, Greece, (nlin/0703037)

13. Voglis N., Kalapotharakos C. and Stavropoulos I., 2002, MNRAS, 337, 619

14. Voglis N., Kalapotharakos C. and Stavropoulos I., 2006, MNRAS, 372, 901

15. Voglis N., Harsoula M. and Contopoulos G, 2007, MNRAS,381, 757

16. Kalapotharakos C., Voglis N. and Contopoulos G., 2004, MNRAS, 428, 905

17. Kalapotharakos C., Efthymiopoulos C. and Voglis N., 2007, (preprint astro$\mathrm{ph} / 0709.0799)$

18. Skokos Ch., Bountis T. and Antonopoulos Ch.,, 2007, Physica D, 231, 30

19. Miyamoto M. and Nagai R., 1975, PASJ, 27, 533

20. Pfenniger D., 1984, A\&A, 134, 373

21. Patsis P. A., Skokos Ch. and Athanassoula E., 2002, MNRAS, 337, 578

22. Patsis P. A., Skokos Ch. and Athanassoula E., 2003a, MNRAS, 342, 69

23. Patsis P. A., Skokos Ch. and Athanassoula E., 2003b, MNRAS, 346, 1031

24. Skokos Ch., Patsis P. A. and Athanassoula E., 2002a, MNRAS, 333, 847

25. Skokos Ch., Patsis P. A. and Athanassoula E., 2002b, MNRAS, 333, 861 